\title{
Erratum to: Cardiac involvement in systemic sclerosis: differences between clinical subsets and influence on survival
}

\author{
Andreu Fernández-Codina ${ }^{1}$ Carmen Pilar Simeón-Aznar ${ }^{1}$ Iago Pinal-Fernandez ${ }^{1}$. \\ José Rodríguez-Palomares $^{2}$ - Maria Nazarena Pizzi ${ }^{3}$ Cristina Eve Hidalgo ${ }^{1}$. \\ Alfredo Guillén-Del Castillo ${ }^{1}$ - Francisco Javier Prado-Galbarro ${ }^{4}$. \\ Antonio Sarria-Santamera ${ }^{4} \cdot$ Vicent Fonollosa-Plà $^{1} \cdot$ Miquel Vilardell-Tarrés ${ }^{1}$
}

Published online: 4 April 2016

(C) Springer-Verlag Berlin Heidelberg 2016

\section{Erratum to: Rheumatol Int \\ DOI 10.1007/s00296-015-3382-2}

The family name of one of the co-authors, Alfredo GuillénDel Castillo, was incorrect in the original publication. The correct family name should read Guillén-Del Castillo.

The online version of the original article can be found under doi:10.1007/s00296-015-3382-2.

Andreu Fernández-Codina

andreu.fernandez@vhebron.net

1 Department of Internal Medicine, Systemic Autoimmune Diseases Unit, Vall d'Hebron University Hospital, Universitat Autònoma de Barcelona, 3rd floot, Hospital General Vall d'Hebron building, Passeig de la Vall d'Hebron 119-129, 08035 Barcelona, Spain

2 Cardiology Department, Vall d'Hebron University Hospital, Universitat Autònoma de Barcelona, Barcelona, Spain

3 Nuclear Medicine Department, Vall d'Hebron University Hospital, Universitat Autònoma de Barcelona, Barcelona, Spain

4 Agencia de Evaluación de Tecnologías Sanitarias, Instituto de Salud Carlos III, Madrid, Spain 